\title{
ON THE BLASCHKE APPROACH OF RULED SURFACE
}

\author{
RASHAD A. ABDEL BAKY
}

\begin{abstract}
In this paper using E. Study map and the Blaschke approach we studied a ruled surface as a curve on the dual unit sphere. The Blaschke approach proceeds by defining a sequence of ruled surfaces associated with the ruled surface. The relative positions of these surfaces and their distribution parameters characterize the local properties of the original surface. A necessary condition for a ruled surface to be closed is derived. Moreover, an example of application is investigated in detail.
\end{abstract}

\section{Introduction}

As a rigid body moves in space lines embedded in the body trace ruled surfaces. These lines may be the axes of the joints of spatial mechanisms or manipulators or the line of action of the end-of-arm tooling of a manipulator. The integral invariants of line trajectories seeks to characterize the shape of the trajectory ruled surface and relate it to the motion of body carrying the line that generates it.

Here the presentation of ruled surfaces is based on work by Blaschke and E. Study [2, $5,9,11]$ from the point of view of the theory of invariants of a transitive transformation group. The fundamental idea is to replace points by lines as fundamental concepts of geometry. Points are then defined by the totality of straight lines passing through them. Oriented lines in a Euclidean three-space $E^{3}$ may be represented by unit vectors with three components over the ring of dual numbers. A differentiable curve on the dual unit sphere depending on one-real parameter " $t$ " corresponds to a ruled surface in $E^{3}$. This correspondence is one-to-one and allows the geometry of ruled surfaces to be represented by the geometry of dual spherical curves on a dual unit sphere. It allows a complete generalization of the mathematical expression for the spherical point geometry to the spatial line geometry by means of dual number extension, i.e. replacing all ordinary quantities by the corresponding dual number quantities $[5,7,11,12]$.

Dual spherical motion, expressed with the help of dual unit vectors, is closely analogous to real shperical motion, expressed with the help of real unit vectors. Therefore, the properties of elementary real spherical motion can also be carried over by analogy into the motion of lines in $E^{3}$.

Received December 10, 2001; revised May 13, 2002.

2000 Mathematics Subject Classification. 53A17.

Key words and phrases. E. Study map, ruled surface, instantaneous screw axis. 
References $[1,2,5,9,11,12]$ contain the necessary basic concepts about the dual elements and one-to-one correspondence between ruled surfaces and one-parameter dual spherical motions.

\section{Basic Concepts}

If $a$ and $a^{*}$ are real numbers, the combination

$$
A=a+\varepsilon a^{*},
$$

is called a dual number. Here $\varepsilon$ is the dual unit. Dual numbers are considered as polynomials in $\varepsilon$, subject to the rules $\varepsilon \neq 0, \varepsilon^{2}=0, \varepsilon \cdot 1=1 . \varepsilon=\varepsilon$. W. K. Clifford defined the dual numbers, the set of dual numbers forms a commutative ring having the numbers $\varepsilon a^{*}$ ( $a^{*}$ real) as divisors of zero, not a field. No number $\varepsilon a^{*}$ has an inverse in the algebra. But the other laws of the algebra of dual numbers are the same as the laws of algebra of complex numbers. For example, two dual numbers $A$ and $B=b+\varepsilon b^{*}$ are added componentwise.

$$
A+B=(a+b)+\varepsilon\left(a^{*}+b^{*}\right),
$$

and they are multiplied by

$$
A B=a b+\varepsilon\left(a^{*} b+a b^{*}\right) .
$$

For the equality of $A$ and $B$ we have

$$
A=B \Leftrightarrow a=b, \quad \text { and } \quad a^{*}=b^{*}
$$

An oriented line in $E^{3}$ may be given by two points $\boldsymbol{x}$ and $\boldsymbol{y}$ on it. If $\mu$ is any non-zero constant, the parametric equation of the line is:

$$
\boldsymbol{y}=\boldsymbol{x}+\mu \boldsymbol{a},
$$

$\boldsymbol{a}$ is a unit vector along the line. The moment of $\boldsymbol{a}$ with respect to the origin is

$$
\boldsymbol{a}^{*}=\boldsymbol{x} \times \boldsymbol{a}=\boldsymbol{y} \times \boldsymbol{a} .
$$

This means that $\boldsymbol{a}$ and $\boldsymbol{a}^{*}$ are not independent of the choice of the points on the line and these vectors are not independent of one another; satisfy the following equations:

$$
<\boldsymbol{a}, \boldsymbol{a}>=1,<\boldsymbol{a}, \boldsymbol{a}^{*}>=0 .
$$

The six components $a_{i}, a_{i}^{*}(i=1,2,3)$ of the vectors $\boldsymbol{a}$ and $\boldsymbol{a}^{*}$ are known to be Plücker homogeneous line coordinates. These two vectors $\boldsymbol{a}$ and $\boldsymbol{a}^{*}$ determine an oriented line in $E^{3}$. A point $\boldsymbol{z}$ lies on this line if and only if

$$
z \times a=a^{*} .
$$


The set of oriented lines in $E^{3}$ is in one-to-one correspondence with pairs of vectors subject to the conditions (2.7), and so we may expect to represent it as a certain fourdimensional set in $\Re^{6}$ of sixtuples of real numbers; we may take the space $D^{3}$ of triples of dual numbers with coordinates:

$$
X_{i}=x_{i}+\varepsilon x_{i}^{*} \quad(i=1,2,3)
$$

Each line in $E^{3}$ may be represented by a dual unit vector

$$
\boldsymbol{A}=\boldsymbol{a}+\varepsilon \boldsymbol{a}^{*}
$$

in $D^{3}$. It is clear that this dual unit vector has the property

$$
<\boldsymbol{A}, \boldsymbol{A}>=<\boldsymbol{a}, \boldsymbol{a}>+2 \varepsilon<\boldsymbol{a}, \boldsymbol{a}^{*}>=1 .
$$

Theorem 2.1. (E. Study) The oriented lines in $E^{3}$ are in one-to-one correspondence with points of the dual unit sphere $\langle\boldsymbol{X}, \boldsymbol{X}\rangle=1$ in $D^{3}[5]$.

By using this correspondence, one can derive the properties of the spatial motion of a line. Hence, the geometry of ruled surface is represented by the geometry of curves on the dual unit sphere in $D^{3}$.

\section{The Blaschke Approach}

A ruled surface is a surface swept out by a stright line $L$ moving along a curve $\boldsymbol{z}=\boldsymbol{z}(t)$. The various position of the generating lines $L$ are called the rulings of the surface. Such a surface, thus, has a parametrization in the ruled form:

$$
\boldsymbol{M}(t, u)=\boldsymbol{z}(t)+u \boldsymbol{a}(t), \quad u \in \Re .
$$

Here $\boldsymbol{z}=\boldsymbol{z}(t)$ is called the base curve, $\boldsymbol{a}=\boldsymbol{a}(t)$ is the unit vector giving the direction of generating line, and $t$ is the motion parameter. The base curve is not unique, since any curve of the form:

$$
\boldsymbol{C}(t)=\boldsymbol{z}(t)+\eta(t) \boldsymbol{a}(t)
$$

may be used as its base curve, $\eta(t)$ is a smooth function. If there exists a common perpendicular to two neighbouring rulings on $\boldsymbol{M}=\boldsymbol{M}(t, u)$, then the foot of the common perpendicular on the main ruling is called a central point. The locus of the central points is called the striction curve. In $(3.2)$ if

$$
\eta(t)=-\frac{<\boldsymbol{z}^{\prime}, \boldsymbol{a}^{\prime}>}{\left\|\boldsymbol{a}^{\prime}\right\|^{2}},
$$

then $\boldsymbol{C}(t)$ is called the striction curve on the ruled surface $\boldsymbol{M}(t, u)$, and it is unique. Here the derivative with respect to $t$ is denoted by a dash over functional symbol. 
The ruled surface $\boldsymbol{M}(t, u)$ corresponds to the dual spherical curve

$$
\boldsymbol{A}(t)=\boldsymbol{a}(t)+\varepsilon \boldsymbol{z}(t) \times \boldsymbol{a}(t)=\boldsymbol{a}(t)+\varepsilon \boldsymbol{a}^{*}(t),
$$

in $D^{3}$. We now define an orthonormal moving frame along this dual curve as follows:

$$
\boldsymbol{A}_{1}=\boldsymbol{A}(t), \quad \boldsymbol{A}_{2}=\frac{\boldsymbol{A}_{1}^{\prime}}{\left\|\boldsymbol{A}_{1}^{\prime}\right\|}, \quad \boldsymbol{A}_{3}=\boldsymbol{A}_{1} \times \boldsymbol{A}_{2} .
$$

From now on we consider the case without $\boldsymbol{a}(t)=$ constant vector and $\boldsymbol{a}^{*}(t)=\mathbf{0}$. In the case $\boldsymbol{a}(t)=$ constant vector the ruled surface $\boldsymbol{M}(t, u)$ is a cylinder and in the case $\boldsymbol{a}^{*}(t)=\mathbf{0}$ the ruled surface $\boldsymbol{M}(t, u)$ is a cone. The frame in (3.4) is called the Blaschke frame. The dual unit vectors $\boldsymbol{A}_{1}, \boldsymbol{A}_{2}$, and $\boldsymbol{A}_{3}$ corresponds to three concurrent mutually orthogonal lines in $E^{3}$. Their point of intersection is the central point on the ruling $\boldsymbol{A}_{1} . \boldsymbol{A}_{3}(t)$ is the limit position of the common perpendicular to $\boldsymbol{A}_{1}(t)$ and $\boldsymbol{A}_{1}(t+d t)$, and is called the central tangent of the ruled surface $\boldsymbol{A}_{1}=\boldsymbol{A}(t)$ at the central point. The line $\boldsymbol{A}_{2}=\boldsymbol{A}_{2}(t)$ is called the central normal of $\boldsymbol{A}_{1}=\boldsymbol{A}(t)$ at the central point. Moreover, the dual planes which correspond to the subspaces $\operatorname{Sp}\left\{\boldsymbol{A}_{1}, \boldsymbol{A}_{2}\right\}, \operatorname{Sp}\left\{\boldsymbol{A}_{3}, \boldsymbol{A}_{2}\right\}$, and $\operatorname{Sp}\left\{\boldsymbol{A}_{1}, \boldsymbol{A}_{3}\right\}$, respectively, are called the tangent plane, asymptotic plane and normal plane. By construction, the Blaschke formula is

$$
\frac{d}{d t}\left(\begin{array}{l}
\boldsymbol{A}_{1} \\
\boldsymbol{A}_{2} \\
\boldsymbol{A}_{3}
\end{array}\right)=\left(\begin{array}{ccc}
0 & P & 0 \\
-P & 0 & Q \\
0 & -Q & 0
\end{array}\right)\left(\begin{array}{l}
\boldsymbol{A}_{1} \\
\boldsymbol{A}_{2} \\
\boldsymbol{A}_{3}
\end{array}\right),
$$

where $P=p+\varepsilon p^{*}=\left\|A_{1}^{\prime}\right\|, Q=q+\varepsilon q^{*}=\frac{\operatorname{det}\left(A, A^{\prime}, A^{\prime \prime}\right)}{P^{2}}$ are called the Blaschke's invariants of the dual curve $\boldsymbol{A}_{1}(t)$. The integrals invariants $\int P d t$, and $\int Q d t$ are the dual arc-length of the dual curves $\boldsymbol{A}_{1}(t)$ and $\boldsymbol{A}_{3}(t)$, respectively. Since $P$ contains only first derivatives of the ruled surface $\boldsymbol{A}_{1}(t)$, it is a first order property of the surface, in particular is its dual speed. Similarly, $Q$ is a second order property of $\boldsymbol{A}_{1}(t)$.

The central point, $\boldsymbol{C}(t)$, satisfies:

$$
\boldsymbol{C} \times \boldsymbol{a}_{i}=\boldsymbol{a}_{i}^{*}, \quad(i=1,2,3) .
$$

The tangent of the striction curve may be wirtten as

$$
\boldsymbol{C}^{\prime}=\alpha \boldsymbol{a}_{1}+\beta \boldsymbol{a}_{2}+\gamma \boldsymbol{a}_{3}
$$

Differentiation of the three equations in (3.6) and using (3.5), we have

$$
\boldsymbol{C}^{\prime}=q^{*} \boldsymbol{a}_{1}+p^{*} \boldsymbol{a}_{3}
$$

The striction curve $\boldsymbol{C}(t)$ is also striction curve of the ruled surface corresponding to the dual curve $\boldsymbol{A}_{3}(t)$. This is shown by calculating it directly from

$$
\boldsymbol{C}_{3}(t)=\boldsymbol{C}(t)-\frac{<\boldsymbol{C}^{\prime}, \boldsymbol{a}_{3}^{\prime}>}{\left\|\boldsymbol{a}_{3}^{\prime}\right\|^{2}} .
$$


Since $\boldsymbol{a}_{3}^{\prime}=-q \boldsymbol{a}_{2}$, it is seen (3.9) that $\boldsymbol{C}_{3}(t)=\boldsymbol{C}(t)$. The two surfaces $\boldsymbol{A}_{1}(t)$ and $\boldsymbol{A}_{3}(t)$ are closely related, each is the central tangent to the other. The first order of one are second order properties of the other, and they have the same central normal $\boldsymbol{A}_{2}$.

The distribution parameters of the ruled surfaces $\boldsymbol{A}_{1}(t), \boldsymbol{A}_{2}(t)$, and $\boldsymbol{A}_{3}(t)$, respectively, are $[5,11]$ :

$$
\lambda_{1}=\frac{p^{*}}{p}, \quad \lambda_{2}=\frac{p p^{*}+q q^{*}}{p^{2}+q^{2}}, \quad \lambda_{3}=\frac{q^{*}}{q} .
$$

\section{Kinematic Interpretation}

The Blaschke invariants $P$, and $Q$ provide a kinematic interpretation of the moving Blaschke frame. To carry out this, we define the dual vector

$$
\boldsymbol{D}=\boldsymbol{d}+\varepsilon \boldsymbol{d}^{*}=Q \boldsymbol{A}_{1}+P \boldsymbol{A}_{3}
$$

known as the Darboux vector of the moving Blaschke frame. This vector allows to collect the Baschke formula by:

$$
\boldsymbol{A}_{i}^{\prime}=\boldsymbol{D} \times \boldsymbol{A}_{i}, \quad(i=1,2,3) .
$$

According to the Darboux vector, at any instant $t$, the dual angular velocity vector of the Blaschke frame with respect to itself has a component $Q$ about $\boldsymbol{A}_{1}$ and $P$ about $\boldsymbol{A}_{3}$. $\|\boldsymbol{D}\|=\sqrt{P^{2}+Q^{2}}=\Omega=\omega+\varepsilon \omega^{*}$ is the angular speed of $\boldsymbol{A}_{1}(t)$ about $\mathbf{D}$;

$$
\omega=\sqrt{p^{2}+q^{2}} \quad \text { and }, \quad \omega^{*}=\frac{p p^{*}+q q^{*}}{\sqrt{p^{2}+q^{2}}} .
$$

are the rotational angular speed and translational angular speed of $\boldsymbol{A}_{1}(t)$, respectively. The pitch of $\boldsymbol{A}_{1}(t)$ along $\boldsymbol{D}$ is $\left(\omega^{*} / \omega\right)$ which is equal to the distribution parameter of $\boldsymbol{A}_{2}(t)$.

We now define the dual unit vector

$$
\boldsymbol{U}(t)=\boldsymbol{u}(t)+\varepsilon \boldsymbol{u}^{*}(t)=\frac{\boldsymbol{D}}{\|\boldsymbol{D}\|}=\frac{Q \boldsymbol{A}_{1}+P \boldsymbol{A}_{3}}{\sqrt{Q^{2}+P^{2}}}
$$

Therefore $\boldsymbol{U}$ is the instantaneous screw axis (I.S.A. for short) of the motion of $\boldsymbol{A}_{1}$ in the Blaschke frame. From equation (3.14), the I.S.A. is parallel to the tangent plane of the ruled surface $\boldsymbol{A}_{1}=\boldsymbol{A}(t)$, and is orthogonal to the central normal $\boldsymbol{A}_{2}$. Let $\Psi=\psi+\varepsilon \psi^{*}$ be the dual angel between the I.S.A. and the ruled surface $\boldsymbol{A}_{1}=\boldsymbol{A}(t)$, then we have that:

$$
\boldsymbol{U}=\cos \Psi \boldsymbol{A}_{1}+\sin \Psi \boldsymbol{A}_{3},
$$

where

$$
\cot \Psi=\frac{Q}{P}
$$

The trigonometric function in (3.16) of $\Psi$ can be expanded as:

$$
\cot \Psi=\cot \psi-\varepsilon \psi^{*}\left(1+\cot ^{2} \psi\right)=\frac{Q}{P}=\frac{q+\varepsilon q^{*}}{p+\varepsilon p^{*}} .
$$


From this equation, we get:

$$
\psi^{*}=\frac{p^{*} q-q^{*} p}{p^{2}+q^{2}},
$$

is the minimal distance from the I.S.A. to the ruled surface $\boldsymbol{A}_{1}(t)$. This distance is measured along the central normal $\boldsymbol{A}_{2}$, and is seen to be the combination of the invariants of ruled surface $\boldsymbol{A}=\boldsymbol{A}_{1}(t)$.

Now, we shall act as we have done in the last. The I.S.A. of the Blaschke frame belonging to the ruled surface $\boldsymbol{U}=\boldsymbol{U}(t)$, generally, generates a ruled surface $\boldsymbol{U}_{1}=\boldsymbol{U}(t)$ which admits the dual frame;

$$
\boldsymbol{U}_{1}=\boldsymbol{U}(t), \quad \boldsymbol{U}_{2}=\frac{\boldsymbol{U}_{1}^{\prime}}{\left\|\boldsymbol{U}_{1}^{\prime}\right\|}, \quad \boldsymbol{U}_{3}=\boldsymbol{U}_{1} \times \boldsymbol{U}_{2},
$$

as the Blaschke frame. Thus, the calculations give that:

$$
\left(\begin{array}{l}
U_{1} \\
U_{2} \\
\boldsymbol{U}_{3}
\end{array}\right)=\left(\begin{array}{ccc}
\cos \Psi & 0 & \sin \Psi \\
-\sin \Psi & 0 & \cos \Psi \\
0 & -1 & 0
\end{array}\right)\left(\begin{array}{l}
\boldsymbol{A}_{1} \\
\boldsymbol{A}_{2} \\
\boldsymbol{A}_{3}
\end{array}\right)
$$

The variations of this frame are analogous to (3.5) and is given by:

$$
\frac{d}{d t}\left(\begin{array}{l}
\boldsymbol{U}_{1} \\
\boldsymbol{U}_{2} \\
\boldsymbol{U}_{3}
\end{array}\right)=\left(\begin{array}{ccc}
0 & \tilde{P} & 0 \\
-\tilde{P} & 0 & \tilde{Q} \\
0 & -\tilde{Q} & 0
\end{array}\right)\left(\begin{array}{l}
\boldsymbol{U}_{1} \\
\boldsymbol{U}_{2} \\
\boldsymbol{U}_{3}
\end{array}\right)
$$

where $\tilde{P}, \tilde{Q}$ are, respectively, the invariants of $\boldsymbol{U}=\boldsymbol{U}_{1}(t) .(3.21)$ can also be written in the form

$$
\boldsymbol{U}_{i}^{\prime}=\tilde{\boldsymbol{D}} \times \boldsymbol{U}_{i}, \quad(i=1,2,3)
$$

where $\tilde{D}$ is the Darboux vector of the frame $\left\{\boldsymbol{U}_{1}, \boldsymbol{U}_{2}, \boldsymbol{U}_{3}\right\}$, i.e.

$$
\tilde{\boldsymbol{D}}=\tilde{Q} \boldsymbol{U}_{1}+\tilde{P} \boldsymbol{U}_{3}
$$

From (3.15) and (3.21), we obtain that:

$$
\boldsymbol{U}_{1}^{\prime}=\Psi^{\prime} \boldsymbol{U}_{2}=\tilde{P} \boldsymbol{U}_{2}
$$

From (3.24), we get the relationship

$$
\tilde{P}=\Psi^{\prime}
$$

Similarly, we have

$$
\tilde{Q}=\sqrt{P^{2}+Q^{2}} .
$$

Comparing (3.11) with (3.23) we observe that the relative velocity of the those Blaschke frames is

$$
\tilde{D}-D=-\Psi^{\prime} \boldsymbol{A}_{2}
$$


Using the Blaschke formula (3.5), the dual curvature function $K(t)=k(t)+\varepsilon k^{*}(t)$, and the dual torsion function $T(t)=\tau(t)+\varepsilon \tau^{*}(t)$ of $\boldsymbol{A}=\boldsymbol{A}_{1}(t)$ are obtained as

$$
\begin{gathered}
K(t)=k(t)+\varepsilon k^{*}(t)=\frac{<\left(\boldsymbol{A}^{\prime} \times \boldsymbol{A}^{\prime \prime}\right),\left(\boldsymbol{A}^{\prime} \times \boldsymbol{A}^{\prime \prime}\right)>}{\left(<\boldsymbol{A}^{\prime}, \boldsymbol{A}^{\prime}>\right)^{2}}=\sqrt{1+\frac{Q^{2}}{P^{2}}} . \\
T(t)=\tau(t)+\varepsilon \tau^{*}(t)=\frac{<\boldsymbol{A}^{\prime},\left(\boldsymbol{A}^{\prime \prime} \times \boldsymbol{A}^{\prime \prime \prime}\right)>}{\left.<\left(\boldsymbol{A}^{\prime} \times \boldsymbol{A}^{\prime \prime}\right),\left(\boldsymbol{A}^{\prime} \times \boldsymbol{A}^{\prime \prime}\right)\right\rangle}=-\frac{d \Psi}{d t} .
\end{gathered}
$$

If $T(t)=\tau(t)+\varepsilon \tau^{*}(t)=0$, i.e. $\psi$ and $\psi^{*}$ are constants, then the line $\boldsymbol{U}$ is fixed to the second order and the line $\boldsymbol{A}$ moves about it with pitch $\lambda_{2}$. Thus locally the ruled surface $\boldsymbol{A}=\boldsymbol{A}_{1}(t)$ is traced during a screw motion of pitch $\lambda_{2}$ about the I.S.A., by the line $\boldsymbol{A}$ located at distance $\psi^{*}$ and angel $\psi$ relative to the I.S.A.. The I.S.A. is called the axis of curvature of the ruled surface $\boldsymbol{A}=\boldsymbol{A}_{1}(t)$, it is analogous to the center of curvature of a curve.

As for the ruled surfaces $\boldsymbol{A}_{1}(t)$ and $\boldsymbol{A}_{3}(t)$, the ruled surfaces $\boldsymbol{U}_{1}(t)$ and $\boldsymbol{U}_{3}(t)$ have the same striction curve and is given by:

$$
\tilde{\boldsymbol{C}}(t)=\boldsymbol{C}(t)+\psi^{*} \boldsymbol{a}_{2}(t) .
$$

Since $\boldsymbol{U}(t)$ does not have a component in the $\boldsymbol{A}_{2}$ - direction. Then the Blaschke frame while moves along the striction curve on the ruled surface $\boldsymbol{A}=\boldsymbol{A}_{1}(t)$, the I.S.A. generates a right helicoid in $E^{3}$. Thus, in general the ruled surface generated by the instantaneous screw axis of the Blaschke frame is a right helicoid whose striction line coincides continously with $\boldsymbol{A}_{2}$.

Now assume that $\boldsymbol{U}(t)$ is a simple closed curve, i.e. $\boldsymbol{U}(t)=\boldsymbol{U}(t+2 \pi)$. In analogy to the geometry of closed space curves [3.7], and ruled surface [10]. The following theorem can be given:

Theorem 3.1. The necessary condition for the I.S.A. generates a closed ruled surface is given by

$$
\int_{t_{0}}^{t} T d t=\int_{t_{0}}^{t}-\left(\frac{d \Psi}{d t}\right) d t=2 \pi n, \quad n \in Z
$$

This last equation says that the integral depends only on the integer $n$. This integral give us that

$$
\Psi(t)=\Psi\left(t_{0}\right)-2 \pi n
$$

Example. Since during the motion of $\boldsymbol{A}_{1}, \boldsymbol{U}$ is rigidly linked to the Blaschke frame $\left\{\boldsymbol{A}_{1}, \boldsymbol{A}_{2}, \boldsymbol{A}_{3}\right\}$, we may choose a dual point $\boldsymbol{X}\left(X_{1}, X_{2}, X_{3}\right)$ in coordinate system $\left\{\boldsymbol{A}_{1}, \boldsymbol{A}_{2}, \boldsymbol{A}_{3}\right\}$ such that whose coordinates

$$
X_{1}=\cos \Theta \sin \Phi, \quad X_{2}=\sin \Theta \sin \Phi, \quad X_{3}=\cos \Phi,
$$

where $\Theta=\theta+\varepsilon \theta^{*}$ and $\Phi=\varphi+\varepsilon \varphi^{*}$. According to the Taylor's series expansion we may write equation (3.33) in the forms:

$$
x_{1}=\cos \theta \sin \phi, \quad x_{2}=\sin \theta \cos \phi, \quad x_{3}=\cos \phi,
$$


and

$$
\left.\begin{array}{l}
x_{1}^{*}=\phi^{*} \cos \theta \cos \phi-\theta^{*} \sin \theta \sin \phi, \\
x_{2}^{*}=\phi^{*} \sin \theta \cos \phi+\theta^{*} \cos \theta \sin \phi, \\
x_{3}^{*}=-\phi^{*} \sin \phi .
\end{array}\right\}
$$

Let $\boldsymbol{y}$ denote the position vector of an arbitrary point $Y\left(y_{1}, y_{2}, y_{3}\right)$ of the line $\boldsymbol{X}$, then

$$
\boldsymbol{x}^{*}=\boldsymbol{y} \times \boldsymbol{x},
$$

or

$$
\left(x_{1}^{*}, x_{2}^{*}, x_{3}^{*}\right)=\left(y_{2} x_{3}-y_{3} x_{2}, y_{3} x_{1}-y_{1} x_{3}, y_{1} x_{2}-y_{2} x_{1}\right) .
$$

Substituting equations (3.34), and (3.35) into (3.36) we have

$$
\left.\begin{array}{l}
y_{2} \cos \phi-y_{3} \sin \theta \sin \phi=\phi^{*} \cos \theta \cos \phi-\theta^{*} \sin \theta \sin \phi, \\
-y_{1} \cos \phi+y_{3} \cos \theta \sin \phi=\phi^{*} \sin \theta \cos \phi+\theta^{*} \cos \theta \sin \phi, \\
\left(y_{1} \sin \theta-y_{2} \cos \theta+\phi^{*}\right) \sin \phi=0 .
\end{array}\right\}
$$

Let

$$
S=\{\boldsymbol{X} ;<\boldsymbol{X}, \boldsymbol{U}>=\cos \Phi=\text { constant, }\|\boldsymbol{X}\|=1\},
$$

be the circle on the dual unit sphere $K$ rigidly connected with $\left\{\boldsymbol{A}_{1}, \boldsymbol{A}_{2}, \boldsymbol{A}_{3}\right\}$. Let $\boldsymbol{X}$ be a point of $S$, then equations of (3.37) have only two real parameters $\theta^{*}$ and $\theta$. So if we choose $\theta^{*}=h \theta, h$ denoting to the pitch of the screw motion, then equations (3.37) represents a ruled surface in $E^{3}$.

We now determine the invariants $P$, and $Q$ of this ruled surface. The derivatives of $\boldsymbol{X}=\boldsymbol{X}(\theta)$ in (3.33) are given by:

$$
\begin{aligned}
& \boldsymbol{x}^{\prime}=\left(\begin{array}{c}
-\sin \theta \sin \phi \\
\cos \theta \sin \phi \\
0
\end{array}\right), \quad \boldsymbol{x}^{\prime \prime}=\left(\begin{array}{c}
-\cos \theta \sin \phi \\
-\sin \theta \sin \phi \\
0
\end{array}\right), \\
& \boldsymbol{x}^{*^{\prime \prime}}=\left(\begin{array}{c}
-\phi^{*} \sin \theta \cos \phi-h \sin \theta \sin \phi-\theta^{*} \cos \theta \sin \phi \\
\phi^{*} \cos \theta \cos \phi+h \cos \theta \sin \phi-\theta^{*} \sin \theta \sin \phi \\
0
\end{array}\right), \\
& \boldsymbol{x}^{*^{\prime \prime}}=\left(\begin{array}{c}
-\phi^{*} \cos \theta-2 h \cos \theta \sin \varphi+\theta^{*} \sin \theta \sin \phi \\
-\phi^{*} \sin \theta \cos \phi-2 h \sin \theta \sin \varphi-\theta^{*} \cos \theta \sin \phi \\
0
\end{array}\right) .
\end{aligned}
$$

The Blaschke invarints are now become

$$
\left.\begin{array}{l}
P=p+\varepsilon p^{*}=\sin \phi\left\{1+\varepsilon\left(\phi^{*} \cot \phi+h\right)\right\}, \\
Q=q+\varepsilon q^{*}=\cos \phi+\varepsilon\left(-\phi^{*} \sin \varphi+h \cos \phi\right) .
\end{array}\right\}
$$

By using the real and dual parts of equations (3.40), then (3.10) becomes

$$
\lambda_{1}=\phi^{*} \cot \phi+h, \quad \lambda_{2}=h, \quad \lambda_{3}=\left(-\phi^{*} \tan \phi+h\right) .
$$


From equaitons $(3.37)$ we have the following cases $\left(\phi^{*} \neq 0\right)$ :

Case 1: If $0<\phi<\pi / 2$, then we have

$$
\left.\begin{array}{l}
y_{2}=\left(y_{3}-\theta^{*}\right) \sin \theta \tan \phi+\phi^{*} \cos \theta, \\
y_{1}=\left(y_{3}-\theta^{*}\right) \cos \theta \tan \phi-\phi^{*} \sin \theta, \\
y_{1} \sin \theta+y_{2} \cos \theta=0 .
\end{array}\right\}
$$

Or

$$
\frac{y_{1}^{2}}{\phi^{* 2}}+\frac{y_{2}^{2}}{\phi^{* 2}}-\frac{Y_{3}^{2}}{\phi^{* 2} \cot ^{2} \phi}=1,
$$

where $Y_{3}=y_{3}-\theta^{*}$. Equation (3.43) represent a one parameter family of one-sheeted hyperboloids. The intersection of each hyperboloid and the corresponding plane $y_{3}=\theta^{*}$ is the circle $y_{1}^{2}+y_{2}^{2}=\phi^{* 2}$. Therefore the envelope of the locus of all lines $\boldsymbol{X}$ in $E^{3}$ is a right circular cylinder.

Hence the following theorem is proved:

Theorem 3.2. During the one-parameter screw motion the locus of the line $\boldsymbol{X}$ in $E^{3}$, which make a cute angel $\phi$ with the I.S.A. of the motion, is a one-parameter family of one sheeted hyperboloids whose envelope is the right circular cylinder $y_{1}^{2}+y_{2}^{2}=\phi^{* 2}$.

Case 2: If $\phi=0$ (or $\pi$ ), then equation (3.37) reduces to

$$
y_{2}=\phi^{*} \cos \theta, \quad y_{1}=-\phi^{*} \sin \theta,
$$

or eliminating the parameter $\theta$ we obtain

$$
y_{1}^{2}+y_{2}^{2}=\phi^{* 2}
$$

which is a one-parameter family of right circular cylinder whose axid is the I.S.A. of the motion.

Hence the following theorem is proved:

Theorem 3.3. During the one-parameter screw motion, the locus of the line $\boldsymbol{X}$ in $E^{3}$, which is parallel to the I.S.A. of the motion, is a right circular cylinder whose axis is the I.S.A. of the motion.

Case 3. If $\phi=\pi / 2$, then equation (3.37) reduces to

$$
y_{3}=\theta^{*}, \quad y_{1} \sin \theta-y_{2} \cos \theta+\phi^{*}=0,
$$

which is a one-parameter family of lines given by the intersection of the planes $y_{3}=\theta^{*}$, and the hyperboliods

$$
\left(y_{1}+\frac{\phi^{*}}{\sin \theta}\right)^{2}=y_{2}^{2} \cot ^{2} \theta,
$$

in this case it is easily seen from (3.41) that the distribution parameter $\lambda_{1}$ is constant.

Hence the following theorem is proved: 
Theorem 3.4. During the one-parameter screw motions the locus of the line $\boldsymbol{X}$ in $E^{3}$, which is orthogonal to the I.S.A. of the motion, is a ruled surface having constant distribution parameter. This ruled surface is the intersection of the planes $y_{3}=\theta^{*}$, and the hyperboliods (3.46).

\section{References}

[1] Abdel Baky, R. A., The relation among Darboux vectors of Ruled Surfaces in a line Congurences, Riv. Mat., Univ. Parma 6(1997), 201-211.

[2] Blaschke, W. Vorlesungen uber Differential Geometrie, Bd 1, Dover Publications, New York, 1945, 260-277.

[3] Fenchel. W., On the Differential Geometry of Closed Space Curves, Bull. AMS 57(1951), 44-54.

[4] Freudenstein, F., Higher path-curvature analysis in plane kinematics, J. of Eng. Ind., Trans of ASME series B 87(1965), 184-190.

[5] Gugenheimer, H. W., Differential Geometery, Mc Graw-Hill, New York, 1956, 162-169.

[6] Harl, J., Zerlegung der Darboux-derhung in zwei ebene drehungen, J. Geom. 47(1993), 32-38.

[7] Huang, C. C., A differential geometric crieterion for a space curve to be closed, Proc. AMS 83(1981), 357-361.

[8] Hoschek, J., Integralinarianten von regelflachen, Arch. Math. V. XXIV, (1973), 218-224.

[9] Muller, H. R., Kinematik Dersleri, T. C. Ankara Univ. Turkey, (1963), 162-169.

[10] Sezer, M., Kose, O, and Nizamoglu, S., A crieterion for a ruled surface to be closed, Doga TR. J. of Math. 14(1990), 39-47.

[11] Veldkamp, G. R., On the use of dual numbers, vectors, and matrices in instantaneous spatial kinematics, Mech. and Mach. Thoery 11(1976), 141-156.

[12] Yang, A. T., Application of Quaternion Algebra and Dual Numbers to the Analysis of Spatial Mechanisms, Doctoral Dissertation, Columbia University, 1963.

Department of Mathematics, Faculty of Science, University of Assiut, 71516, Assiut, Egypt. 